\title{
Consideraciones éticas relacionadas al manejo de muestras de investigación en cáncer
}

\author{
Ethical considerations related to the management of cancer research samples \\ Carlos A. Castañeda ${ }^{1, a, b, c}$ Miluska Castillo ${ }^{1, d}$, Jule Vásquez ${ }^{1, a}$, Luis A. Bernabe ${ }^{1, d}$, Henry L. Gomez ${ }^{1, a, e, f}$, \\ Gustavo Sarria-Bardales ${ }^{1, \mathrm{~g}}$, Juvenal Sanchez ${ }^{1, \mathrm{f,h}}$.
}

\section{RESUMEN}

El análisis molecular de material biológico humano permite la identificación de futuros biomarcadores para mejorar el manejo de pacientes con cáncer. Sin embargo, el manejo de estas muestras requiere consideraciones éticas particulares. Distintas organizaciones como el Consejo Europeo y agencias de gobierno de Estados Unidos han generado distintos documentos con definiciones, mecanismos y reglamentos para evitar poner en riesgo de daño o vulnerar los derechos de los donantes de muestras biológicas. Finalmente, todos estos documentos han evolucionado en el tiempo y han permitido que las instituciones de investigación cuenten con comités y regulaciones en ética estándares. Así, las instituciones legales puedan crear precedentes y generar sentencias coherentes.

PALABRAS CLAVE: Ética, manejo de especímenes, biomarcadores de tumor, neoplasias. (Fuente: DeCS BIREME)

\section{SUMMARY}

Molecular analysis of human biological material allows the identification of future biomarkers to improve the management of patients with cancer. However, the handling of these samples requires particular ethical considerations. Different organizations such as the European Council and government agencies of the United States have generated different documents with definitions, mechanisms, and regulations to avoid putting at risk of harm or violating the rights of donors of biological samples. Finally, all these documents have evolved over time and have allowed research institutions to have standard committees and regulations in ethics. Thus, legal institutions can create precedents and generate coherent sentences.

KEYWORDS: Ethics, specimen handling, biomarkers, tumor, neoplasms. (Source: MeSH NLM)

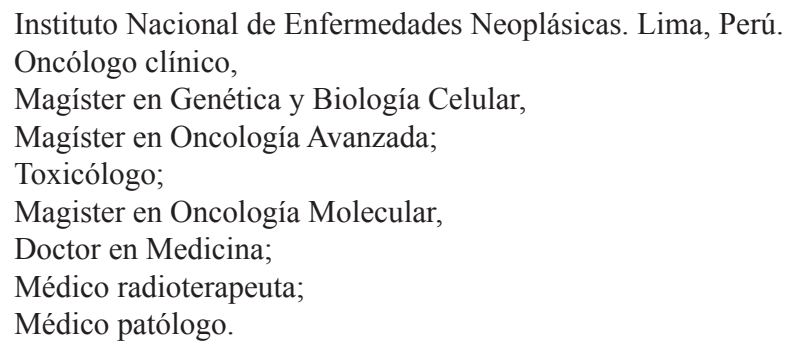




\section{INTRODUCCIÓN}

El desarrollo de la medicina personalizada exige una mejor comprensión de la biología tumoral y la identificación de biomarcadores en cáncer; y ha requerido la investigación en muestras biológicas humanas. Los biomarcadores incluyen el análisis de expresión proteica, expresión génica, y de mutaciones somáticas y heredadas que son medidas objetivamente e indican procesos biológicos normales, procesos patológicos o respuesta farmacológica a intervenciones terapéuticas. Los resultados de las pruebas genéticas/ genómicas permiten identificar a individuos específicos y pueden revelar información acerca del individuo y en algunos casos de los miembros de sus familias (susceptibilidad actual y futura a enfermedades). Los biomarcadores son componentes clave de los estudios translacionales, e idealmente el desarrollo de terapias dirigidas debe estar estrechamente vinculada con la identificación y validación de biomarcadores (1).

El objetivo del artículo fue revisar las consideraciones éticas relacionadas al manejo de muestras de pacientes con cáncer en investigación, incluye también diversos conceptos y ejemplos de investigación no oncológica que se pueden extrapolar a la esfera oncológica.

\section{Muestras biológicas para diagnóstico (MBD)}

Se entiende como muestra biológica a cualquier material biológico de origen humano que pueda albergar información sobre la dotación genética característica de una persona; que incluye: sangre $\mathrm{y}$ sus derivados, lesiones tumorales u órganos y sus remanentes susceptibles de conservación. Adicionalmente, la "Convención para la Protección de los Derechos Humanos y la Dignidad del Ser Humano con Respecto a la Aplicación de la Biología y la Medicina: Convención sobre Derechos Humanos y Biomedicina" del Consejo Europeo (CE) especifica que la denominación "partes del cuerpo" se aplica a "órganos y tejidos propiamente dichos, incluida la sangre", pero "no a productos como cabello y uñas, que son tejidos descartados, y cuya venta no es una afrenta a la dignidad humana" (2). La directiva 2004/23/CE del Parlamento y CE, define además el término "donante" para designar toda fuente humana, ya sea viva o fallecida, de células o tejidos humanos (3).

Los resultados de las pruebas clínicas realizadas en MBD son reportadas formalmente en la historia clínica, y debe tener una utilidad y costo razonable (tabla 1). La realización de pruebas clínicas en muestras tomadas no requiere la firma de un consentimiento informado (CI) específico, aunque este si es necesario al obtener la muestra debido a los riesgos en su obtención. El estudio de mutaciones genéticas hereditarias que requiere además consejo genético previo como genes BRCA1/2 son la excepción que si requiere CI (4).

Adicionalmente existe la aprobación del derecho de propiedad sobre el tejido removido. Las MBD almacenadas en bloques de parafina que fueron parte de diagnósticos como malignidad son consideradas parte de la historia médica y requieren permanecer en los archivos de patología en la mayoría de instituciones. Este material permite realizar revisiones tipo segundaopinion, permite obtener información adicional para casos médico-legales, y permite realizar estudios de biomarcadores estándares que se pudieran desarrollar en el futuro. Sin embargo, los donantes pueden retener algunos derechos de propiedad en ciertos tejidos como los embriones, óvulos, esperma, y órganos con fines de trasplante, y las instituciones se comportan como los custodios legales (5).

El artículo 21 de la Convención de Oviedo (CO), declara: "El cuerpo humano y sus partes no deberán, en sí mismos, dar lugar a un beneficio económico". Mientras que el artículo 22 declara: "Cuando en el curso de una intervención se retira cualquier parte de un cuerpo humano, puede almacenarse y utilizarse para un fin distinto de aquel para el que fue eliminado, solo si esto se hace de conformidad con la información apropiada y los procedimientos de CI" (6).

Por ello, Beyleveld y col. (7), mencionan que el derecho a utilizar el tejido extraído en actividades distintas al diagnóstico como propósitos de investigación de biomarcadores puede interpretarse en forma literal y requerir un $\mathrm{CI}$ específico, o no requerirlo si no producen ganancia económica ni afecta la susceptibilidad del donante. En este mismo sentido de ausencia de riesgo o daño a los donantes en el análisis de MBD, el código de regulaciones federales (CFR del inglés Code of Federal Regulations) de los EEUU para la protección de sujetos humanos en investigación (8), en el Titulo 45 (CFR 45:46) indica que la investigación que se realiza en MBD no requieren cumplir las regulaciones de protección de sujetos humanos en investigación.

El Consejo Europeo a través de la Convención de Oviedo y sus 4 protocolos adicionales indica: "los 
intereses y el bienestar del ser humano en relación a las pruebas genéticas prevalecen sobre los intereses aislados de la sociedad o la ciencia", Los principios de no discriminación y estigmatización del artículo 4 establecen: "Se prohíbe que cualquier forma de discriminación contra una persona, como individuo o como miembro de un grupo en el campo de su herencia genética. Se deben tomar medidas apropiadas para prevenir la estigmatización de personas o grupos en relación a características genéticas". Establecen claramente que toda persona tiene el derecho de conocer cualquier información obtenida sobre su salud (2).

Así mismo, el artículo 4 del reglamento de la Ley peruana 29414 en el Capítulo II, Subcapítulo II, relacionado al acceso a la información indica que debe existir un CI para divulgar la información de la historia clínica o del estado general de los pacientes atendidos (9). La directiva de protección de datos de la Unión Europea (95/46/CE) impone en su artículo 8, un nivel alto de protección a la información de salud y considera que se puede compartir para investigación en escenarios en que se cuente con anonimización total o cuente con un CI de aceptación explícito del propósito de obtener la MBI (10).

Diversos formatos institucionales deben ser desarrollados a través de reuniones de miembros del departamento de patología, comité revisor de proyectos de investigación (con siglas IRB en inglés, de Institutional Review Board), el comité de ética en investigación (CEI) y representantes legales de la institución, para permitir que los departamentos involucrados en el manejo de la neoplasia puedan realizar investigaciones en $\mathrm{MBD}$, generar colaboraciones con laboratorios de investigación externos, trazar constantemente la ubicación de estas y asegurar que las MBD no sean agotadas en pruebas de investigación puesto que pueden ser requeridas para pruebas diagnósticas que se incorporen en el futuro. Estos formatos deben incorporar a los donantes en la decisión de permitir realizar investigación en el material remanente (11).

\section{Muestras biológicas obtenidas para investigación (MBI)}

Las pruebas de laboratorio de investigación no han sido aprobadas por los cuerpos de acreditación para uso clínico y sus resultados pueden o no ser reportados, no deberían ser parte de la historia clínica, y no debe cobrarse a los donantes (tabla 1) (12).
Los ensayos clínicos en cáncer incorporan la toma de biopsias de MBI únicas o múltiples cada vez con mayor frecuencia. Las biopsias para obtener muestras biológicas para investigación (MBI) pueden ser obligatorias $\mathrm{u}$ opcionales dependiendo de lo especificado en el proyecto (13). El riesgo de complicaciones moderada/severas al obtener biopsias de MBI varía según la localización y es de menos de $1 \%$ para órganos sólidos intraabdominales y $2,4 \%$ para biopsias intratorácicos (14).

Los IRB/CEI revisan y aprueban los proyectos de investigación luego de establecer que el riesgo para los participantes sea razonable en relación con el beneficio obtenido con el conocimiento generado. El beneficio de obtener MBI puede ser seleccionar o randomizar al donante (biomarcadores integrados), o la generación de conocimiento (ciencia correlativa) que incluye la obtención de dosis máxima tolerada para el desarrollo del fármaco en los siguientes ensayos clínicos y confirmación del mecanismo de acción y resistencia del fármaco, el objetivo o ambos (tabla 1).

El concepto de aceptar un riesgo personal para beneficio científico ha sido evaluado en los códigos de ética más importantes como la Declaración de Helsinki y se acepta como éticamente aceptable (15). Desafortunadamente, la información producida no siempre logra probar la hipótesis y tener una utilidad, como lo indica Sweis y col. (16), quienes encontraron que solo 12 de 72 ensayos clínicos fase 1 con análisis de biomarcadores de farmacodinamia en MBI lograron identificar un biomarcador significativamente asociado al objetivo buscado. La obtención del beneficio de generar conocimiento requiere informar los resultados del análisis de las MBI en la literatura científica a través de publicaciones. Parseghian et al. (17), encontraron que solo 18 (39\%) de 46 ensayos clínicos con análisis de MBI realizadas entre 2005 y 2010 en el MD Anderson Cancer Center informaron resultados derivados de las biopsias de investigación, a pesar de que $36(78 \%)$ ensayos publicaron al menos un informe del ensayo clínico. Este problema es enfrentado con las regulaciones recientes del Departamento de Salud y Servicios Humanos de los EEUU que exigen no solo el registro de ensayos clínicos sino también el informe de los resultados (18).

El CFR45:46 establece que los participantes de la investigación deben asentir el uso de las MBI en una investigación distinta a la que asintió inicialmente a través de un CI específico, luego de brindarse información adicional suficiente. Sin embargo, existen 
Tabla 1. Tipos de muestras biológicas y sus funciones en la atención médica del paciente y en la investigación.

Tipo de muestra biológica $\quad$ Función en la atención médica del paciente $\quad$ Función en la investigación

\begin{tabular}{|c|c|c|}
\hline $\begin{array}{l}\text { Muestra Biológica } \\
\text { Diagnóstica }\end{array}$ & $\begin{array}{l}\text { Los resultados se usarán directamente } \\
\text { para guiar la atención médica del } \\
\text { paciente sometido al procedimiento de } \\
\text { toma de muestra. }\end{array}$ & $\begin{array}{l}\text { Parte de la muestra puede utilizarse para } \\
\text { investigación o se puede almacenar para } \\
\text { futuras investigaciones potenciales. }\end{array}$ \\
\hline \multirow{3}{*}{$\begin{array}{l}\text { Muestra de investigación } \\
\text { para ciencia correlativa }\end{array}$} & $\begin{array}{l}\text { Los resultados tienen que ser incluidos } \\
\text { en la historia clínica. }\end{array}$ & $\begin{array}{l}\text { Los resultados no requieren colocarse en la } \\
\text { historia clínica. }\end{array}$ \\
\hline & $\begin{array}{l}\text { Este tipo de muestra establece asociación } \\
\text { entre característica molecular del tumor } \\
\text { y característica clínica. El beneficio } \\
\text { directo para los donantes no está } \\
\text { establecido. }\end{array}$ & $\begin{array}{l}\text { La muestra biológica se usará para evaluar } \\
\text { la correlación entre las características } \\
\text { moleculares del tejido y el tipo de } \\
\text { enfermedad del paciente o los efectos del } \\
\text { tratamiento }\end{array}$ \\
\hline & $\begin{array}{l}\text { Los resultados no se utilizarán para } \\
\text { afectar el cuidado del participante de la } \\
\text { investigación. }\end{array}$ & $\begin{array}{l}\text { El propósito de la investigación puede ser } \\
\text { exploratorio (empleado para identificar } \\
\text { posibles nuevos biomarcadores) o } \\
\text { predefinido (destinado a evaluar o validar } \\
\text { uno o más biomarcadores conocidos). }\end{array}$ \\
\hline \multirow[t]{2}{*}{$\begin{array}{l}\text { Muestra de investigación } \\
\text { para estudio de } \\
\text { biomarcadores integrales }\end{array}$} & $\begin{array}{l}\text { El beneficio directo para los donantes no } \\
\text { está establecido. }\end{array}$ & $\begin{array}{l}\text { La muestra se usará para determinar si un } \\
\text { paciente es elegible para un ensayo clínico o } \\
\text { para evaluar uno o más biomarcadores que se } \\
\text { usarán para guiar la intervención dentro del } \\
\text { ensayo. }\end{array}$ \\
\hline & $\begin{array}{l}\text { Los resultados se usarán para decidir } \\
\text { directamente el tratamiento que recibirá } \\
\text { el paciente dentro de un ensayo clínico }\end{array}$ & $\begin{array}{l}\text { Uno de los propósitos del ensayo es evaluar } \\
\text { o validar los biomarcadores. }\end{array}$ \\
\hline
\end{tabular}

algunos CI más amplios que frecuentemente son utilizados en muestras obtenidas para biobancos, pero estos debieran limitarse a una sola disciplina, como el caso de la oncología (10).

La consideración de pertenencia para MBI es igualmente confusa. Adicionalmente, se incluye el término "custodia" para la responsabilidad de cuidar las MBI considerando un almacenamiento seguro, así como el manejo de tejidos extraídos para fines específicamente autorizados por los donantes y asegurando la privacidad de los participantes de acuerdo a las recomendaciones del Instituto de Cáncer de los EEUU (19).

Un caso legal representativo es el de Dr. Catalona vs la Universidad de Washington (UW), en que la corte en este caso, concluyó que la Universidad donde se realiza la investigación es a la que le pertenecen las MBI. Dr. Catalona estableció un gran bio-repositorio de cáncer de próstata en la UW, y cuando cambió de lugar de trabajo, envió una carta con un formulario a sus pacientes para que soliciten la re-ubicación de sus muestras; cerca de 6000 pacientes firmaron y regresaron la forma. La UW rechazó cumplir con lo requerido y solicitó establecer la pertenencia. La corte concluyó que las muestras pertenecían a UW, no a los donantes o al Dr. Catalona y que los donantes donadores de tejidos no mantenían interés de pertenencia sobre el material genético. Si los donantes retiraban sus CI para el proyecto, la Universidad estaba autorizada a destruirlas, anonimizarla o continuar almacenándolas (sin utilizarlas en investigación) (20).

Se concluye, que el manejo de $\mathrm{MBI}$ residuales que se utilizarán en investigación oncológica y las que se toman con el objetivo propio de investigar deben producir un beneficio superior al riesgo y no producir vulneración sobre los donantes oncológicos.

\section{Agradecimiento:}

Este trabajo fue financiado por CONCYTEC (contrato: 198-2015-FONDECYT).

\section{Contribución de autoría:}

CAC y MCG participaron en la concepción, el diseño y la redacción del artículo; HLG, JV, GSB, JS y LAB participaron en la revisión crítica del artículo; y todos los autores realizaron la aprobación de su versión final. 


\section{Declaración de conflictos de interés:}

Los autores declaran no tener conflictos de interés

\section{Correspondencia:}

Carlos A. Castañeda

Av. Angamos este 2520 Surquillo, Lima,Perú.

Teléfono: 51992157220

Correo electrónico: carloscastanedaaltamirano@ yahoo.com

\section{REFERENCIAS BIBLIOGRÁFICAS}

1. European Society for Medical Oncology. ESMO Factsheets on Biomarkers. Lugano: ESMO; 2018. (citado el 10 agosto del 2018) Disponible en: https:// oncologypro.esmo.org/Education-Library/ Factsheets-on-Biomarkers

2. Council of Europe. Details of Treaty No.164: Convention for the Protection of Human Rights and Dignity of the Human Being with regard to the Application of Biology and Medicine: Convention on Human Rights and Biomedicine. trasbourg Cedex: Council of Europe; 1999. (citado el 10 agosto del 2018) Disponible en: http://conventions.coe.int/ Treaty/EN/Reports/Html/164.htm

3. Parlamento Europeo; Consejo De La Unión Europea. Directiva 2004/23/CE del Parlamento Europeo y del Consejo, de 31 de marzo de 2004, relativa al establecimiento de normas de calidad y de seguridad para la donación, la obtención, la evaluación, el procesamiento, la preservación, el almacenamiento y la distribución de células y tejidos humanos. Estrasburgo: Diario Oficial de la Unión Europea; 7 de abril del 2004.

4. Ministerio de Salud. Decreto Supremo 027-2015-SA. Reglamento de la Ley 29414 que establece los derechos de las personas usuarias de los Servicios de Salud. Lima: Diario Oficial El Peruano;13 de agosto de 2015.

5. Epstein JI. Who is the owner of the slides, blocks and fixed tissues? Am J Surg Pathol. 2002; 26:274.

6. Council of Europe Convention of Biomedicine. Convention for the protection of human rights and dignity of the human being with regard to the application of biology and medicine: convention on human rights and biomedicine (adopted by the Committee of Ministers on 19 November 1996). Hum Reprod. 1997; 12(9):2076-80.

7. Beyleveld D, Brownsword R. My body, my body parts, my property? Health Care Anal. 2000; 8(2):8799.

8. U.S. Department of Health \& Human Services. Code of Federal Regulations: Title 45; Public Welfare; Department of Health and Human Services PART 46; Protection of human subjects. Washington
DC: U.S. Department of Health \& Human Services; 2009.

9. Ministerio de Salud. Reglamento de La Ley $\mathrm{N}^{\circ}$ 29414, Ley que establece los derechos de las personas usuarias de los servicios de salud. Lima: MINSA; 2015.

10. European Parliament. Directive E. 95/46/EC of the European Parliament and of the Council of 24 October 1995 on the protection of individuals with regard to the processing of personal data and on the free movement of such data. Official Journal L. 1995; 281:0031- 0050.

11. Hakimian R, Taube S, Bledsoe M, et al. National Cancer Institute Cancer Diagnosis Program: 50-State Survey of Laws Regarding the Collection, Storage, and Use of Human Tissue Specimens and Associated Data for Research. Bethesda, MD: National Institutes of Health; 2004.

12. Department of Health and Human Services. 42 CFR $\S 411.15$ - Particular services excluded from coverage. Washington: Office of the Federal Register National Archives and Records Administration; 1987

13. Peppercorn J, Shapira I, Collyar D, et al. Ethics of mandatory research biopsy for correlative end points within clinical trials in oncology. J Clin Oncol. 2010; 28(15):2635-40.

14. Overman MJ, Modak J, Kopetz S, et al. Use of research biopsies in clinical trials: are risks and benefits adequately discussed? J Clin Oncol. 2013; 31(1):17-22.

15. Asociación Médica Mundial. Declaración de Helsinki de la AMM - Principios éticos para las investigaciones médicas en seres humanos. Helsinki: Asociación Médica Mundial; 2009.

16. Sweis RF, Drazer MW, Ratain MJ. Analysis of impact of post-treatment biopsies in phase I Clinical Trials. J Clin Oncol. 2016; 34(4):369-74.

17. Parseghian CM, Raghav K, Wolff RA, et al. Underreporting of research biopsies from clinical trials in oncology. Clin Cancer Res. 2017; 23(21):6450-7.

18. Zarin DA, Tse T, Williams RJ, Carr S. Trial Reporting in ClinicalTrials.gov - The Final Rule. N Engl J Med. 2016; 375(20):1998-2004.

19. Harty-Golder B. Retention and ownership of blocks. Med Lab Observ. 2004; 36:37.

20. FindLaw's Legal Professional. Washington University vs Catalona. Northern California: FindLaw's Legal Professional; 2007. (Fecha de acceso: 10 agosto del 2018) Disponible en: https:// caselaw.findlaw.com/us-8th-circuit/1300306.html

Recibido: 30/03/2019

Aceptado: 28/06/2019 